\title{
Frequency and phase relations of entangled photons observed by a two-photon interference experiment
}

\author{
Joakim Bergli, ${ }^{*}$ Guillaume Adenier, Andreas P. Thörn, and Arnt Inge Vistnes \\ Department of Physics, University of Oslo, Sem Salands vei 24, 0316 Oslo, Norway
}

(Received 16 May 2019; published 30 August 2019)

\begin{abstract}
An entangled photon experiment has been performed with a large variation of the temperature of the nonlinear crystal generating the entangled pair by spontaneous downconversion. The photon pairs are separated by a nonpolarizing beamsplitter, and the polarization modes are mixed by half-wave plates. The correlation function of the coincidences is studied as a function of the temperature. In the presence of a narrow interference filter, we observe that the correlation changes between -1 and +1 about seven times within a temperature interval of about $30^{\circ} \mathrm{C}$. We show that the common simplified single-mode pair representation of entangled photons is insufficient to describe the results, but that the biphoton description that includes frequency and phase details gives a close to perfect fit with experimental data for two different choices of interference filters. We explain the main ideas of the underlying physics, and we give an interpretation of the two-photon amplitude, which provides an intuitive understanding of the effect of changing the temperature and inserting interference filters.
\end{abstract}

DOI: 10.1103/PhysRevA.100.023850

\section{INTRODUCTION}

One of the central experiments in quantum optics is the Hong-Ou-Mandel (HOM) two-photon interference experiment [1]. In the original version, two entangled photons with the same polarization were generated by downconversion and directed on a beamsplitter from opposite sides. With the timing adjusted so that the two photons came to the beamsplitter at the same time, it was observed that the two photons always exited in the same direction. This could then be interpreted as the interference of two independent photons, where the coalescence of the two photons was seen as a consequence of the bosonic nature of photons. However, similar experiments were soon devised in which the two photons reached the beamsplitter at different times [2], where they were distinguishable by different polarizations [3], or they did not even have to meet in a beamsplitter [4]. It became clear that the proper way of interpreting these experiments had to be in terms of interference between two different two-photon states. For a more complete discussion of the different experiments and a review of the literature, see Ref. [5].

The state of the field after the downconversion process is well known [6-12] and consists of a superposition of many frequency modes; a brief derivation is given below. However, in many situations (see, for instance, [6-12]) one can simplify the description to a single frequency mode and two orthogonal polarizations. Nevertheless, there are cases in which this is insufficient, and the spread in frequencies is important for the understanding of experiments [13,14]. As demonstrated by Fedrizzi et al. [13], one can reveal what they call "hidden entanglement," that is, the state is indeed frequency-entangled. This is done by changing the temperature of the nonlinear crystal where the photon downconversion takes place. The efficiency of the downconversion process

\footnotetext{
*joakim.bergli@fys.uio.no
}

is roughly identical over a rather broad temperature range. However, the mean energies of the two photons are in general different from each other for most of this range. Only in a rather narrow optimal temperature range are the energies of the two outgoing photons equal (on average). We present an experimental setup that allows essentially all possible correlation measurements to be performed in a two-photon interference setup. With this we study how the correlations between the two photons vary when the temperature of the nonlinear crystal is varied. We can then observe the frequency entanglement as demonstrated in Ref. [13] but in a much more dramatic way. Using an interference filter, we restrict the number of frequency modes available, and we investigate to what extent we can recover the predictions of the simplified, single-frequency mode description. We find that at the optimal temperature of the nonlinear crystal, the single frequency mode is always applicable, and we identify "difficult" temperatures, where extremely narrow filters would have to be used in order to restore the single frequency picture (or alternatively, where the frequency entanglement produces pronounced effects, even if only a narrow range of frequencies is involved). We provide a theoretical description that reproduces all experimental results, and we show how it can be used to understand why frequency entanglement is irrelevant at the optimal temperature, and why it becomes relevant at other temperatures. A preliminary report of our experiments is available in [15], where more experimental results are given. Here we reproduce only those results that are directly relevant to our discussion, and we provide a detailed theoretical analysis of the results.

\section{EXPERIMENTAL SETUP}

Our source of polarization-entangled photons (see Fig. 1) is directly inspired by the setup implemented by Kuklewicz et al. [16]. The pairs of photons are obtained by type-II spontaneous parametric downconversion in a periodically poled crystal 


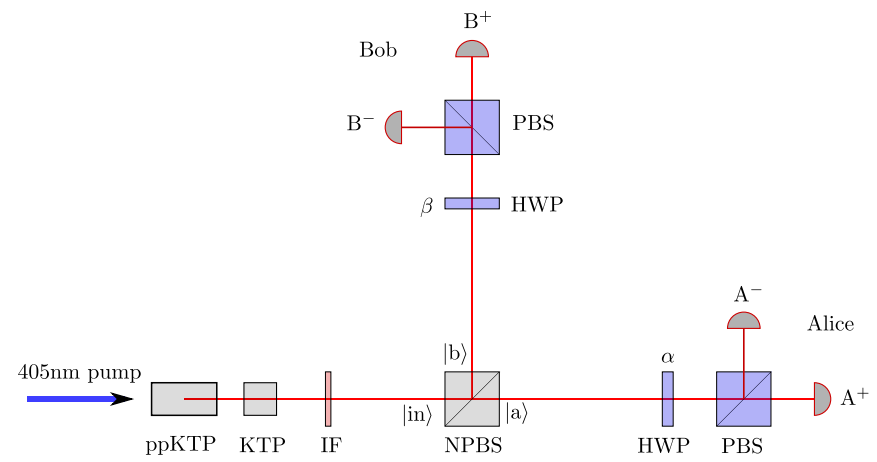

FIG. 1. Experimental setup. The pairs of orthogonally polarized photons are downconverted from the $405 \mathrm{~nm}$ pump in the ppKTP crystal. They are time-compensated (in a KTP crystal), frequencyfiltered (IF), and spatially filtered (iris) before being dispatched to Alice and Bob by a nonpolarizing beamsplitter (NPBS). At each measuring station, a half-wave plate (HWP) rotates the polarization of the photons, and a PBS projects them to a fixed basis. The output of the PBS feeds four detectors: $A^{+}$and $A^{-}$for Alice, $B^{+}$and $B^{-}$for Bob.

of potassium-titanyl-phosphate (ppKTP), under quasi-phasematching (QPM) condition. The pump, a continuous-wave laser at $405 \mathrm{~nm}$, and the downconverted photons are all collinear. The temperature of the ppKTP crystal is controlled by a thermoelectric Peltier temperature controller.

After filtering out the pump with a series of dichroic and interference filters (IFs) (in particular one with a bandwidth of $1 \mathrm{~nm}$ ), the collinear downconverted photons are dispatched by a 50:50 nonpolarizing beamsplitter (NPBS) to two polarization analyzers. We label the polarization analyzer in the transmitted beam as "Alice," and the one in the reflected beam as "Bob." Each consists of a half-wave plate that rotates the polarization of the field, with an angle $\alpha$ for Alice and $\beta$ for Bob, followed by a polarizing beamsplitter (PBS) that projects it in a fixed basis $\{|H\rangle,|V\rangle\}$.

The outputs of each PBS are feeding two detectors, labeled $A^{+}$and $A^{-}$for Alice (respectively located at the transmitted and reflected output of Alice's PBS) and $B^{+}$and $B^{-}$for Bob (respectively located at the transmitted and reflected output of Bob's PBS). The detectors are four avalanche photodiodes SPCM-AQRH-16, from Perkin-Elmer, with a detection efficiency specified at $60 \%$ by the manufacturer with 25 dark counts per second. The detection events (clicks) are timetagged with nominal picosecond precision by a Multichannel Picosecond Event Timer (Hydraharp 400, from Picoquant), and saved to disk for on-the-fly analysis (when the data flow is not too important), and also for subsequent analysis.

The flexibility of this acquisition setup based on the recording of the detection time of the photons is of course largely inspired by the landmark Innsbruck experiment performed by Weihs et al. [17].

Unless specified otherwise, the acquisition duration for each measured point lasted precisely one second, so that the number of counts recorded during this interval can be used as an estimate of the rate of the corresponding counts.

All detected events are recorded with the name of the detector that fired and the time associated with the detection event. No detected events are discarded, so that the coincidence analysis can be performed after the fact with adjustable

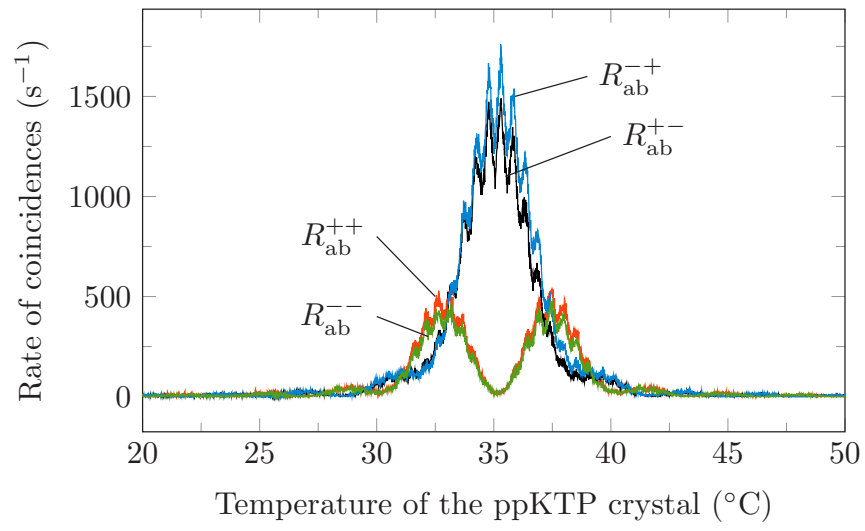

FIG. 2. Rates of coincidences in the diagonal bases $(\alpha=\beta=$ $\pi / 8$ ) as a function of the temperature of the ppKTP crystal. The rate of coincidences drops quickly away from the optimal temperature because the distribution of the wavelengths of the idler and signal is no longer centered on the $810 \mathrm{~nm}$ of our narrow bandwidth interference filter. The rapid oscillations in the coincidence rates arise from the reflections of the pump beam from the ends of the ppKTP crystal, which gives alternatingly constructive and destructive interference between the direct and reflected beams, and an effective oscillation in the pump amplitude. These oscillations are not interesting to us, and they are absent from the correlations as described by Eq. (1) since an overall intensity is normalized out in calculations of correlations.

parameters (size of the coincidence window and timingoffset). Having a complete record of all the detected events allows us to be thorough in the coincidence analysis: we can naturally measure several type of rates, including the rate of single counts, of course, but also the rate of coincidences, the rate of double-counts, or even the triple-counts.

A rate of coincidences denotes the number of times one of Alice's detectors triggers within the same time window (of arbitrary width) as one of Bob's detectors, during an acquisition of $1 \mathrm{~s}$. The four possible rates of coincidences between Alice's and Bob's detectors are denoted $R_{a b}^{++}, R_{a b}^{+-}$, $R_{a b}^{-+}$, and $R_{a b}^{--}$, where the first superscript index indicates which of Alice's detectors is considered, and the second which of Bob's detectors is considered.

A rate of double-counts denotes the number of times the two detectors located on the same side (Alice's side or Bob's side) are both triggered within the same time window, during an acquisition of $1 \mathrm{~s}$. The rate of double-counts measured by Alice is denoted $R_{a a}^{ \pm}$; that measured by Bob is denoted $R_{b b}^{ \pm}$.

\section{EXPERIMENTAL RESULTS}

For the downconversion process, there is an optimal temperature of the ppKTP crystal (in our case $35.1^{\circ} \mathrm{C}$ ), where the frequencies of the two downconverted photons are the same. We study the properties of our entangled photons when the temperature of the ppKTP crystal is brought away from this optimal temperature.

In Fig. 2 we show the coincidence rates as functions of the ppKTP temperature for the case in which the axes of the HWPs are set to $\alpha=\beta=\pi / 8$, which means that both horizontally and vertically polarized photons are rotated into equal superpositions of horizontal and vertical polarization, as 
described by Eq. (13) below. We refer to this as a measurement in the diagonal bases.

The first noticeable effect of bringing the temperature of the ppKTP crystal away from the optimal temperature is that the rate of detected singles and coincidences quickly drops, as can be seen for the coincidences in Fig. 2. The reason for this behavior is that the spectrum of the downconverted photons depends on the temperature of the ppKTP crystal. Indeed, all the terms in the quasi-phase-matching condition of Eq. (4) depend on the temperature of the ppKTP crystal $[18,19]$. At the optimal temperature, both outgoing beams have the same frequency, corresponding to a wavelength $\lambda=810 \mathrm{~nm}$, exactly twice that of the pump beam. As the temperature deviates from the optimal, the center frequencies of the two beams start to differ, while keeping the sum constant in accordance with the energy conservation condition [Eq. (3) below]. Now, because of the interference filter with a narrow bandwidth of $1 \mathrm{~nm}$ in our setup (see Fig. 1), we are nevertheless selecting those pairs of photons that happen to have the same wavelength of $810 \mathrm{~nm}$. As the temperature is changed away from the optimum temperature, the pairs of photons that match this strict wavelength criterion are less and less frequent, so that the number of coincidences drops quickly.

Even though the number of pairs that passes through diminishes away from the optimal temperature, we can still measure their coincidences and their correlation. The correlation

$$
E_{a b}(\alpha, \beta)=\frac{R_{a b}^{++}-R_{a b}^{+-}-R_{a b}^{-+}+R_{a b}^{--}}{R_{a b}^{++}+R_{a b}^{+-}+R_{a b}^{-+}+R_{a b}^{--}}
$$

is a linear combination of the coincidence rates normalized by the sum of coincidences rates. It is therefore quite insensitive to fluctuations in the total rate of detected pairs. Reducing the number of detected pairs decreases the statistical accuracy of the measured correlation, but it does not change this correlation per se. It can be compensated simply by increasing the power of the pump accordingly, which we have done in some of the experimental runs reported below when the rate of detected pairs was too low.

Quite generally, a direct way to assess and fine-tune the quality of the produced polarization-entanglement is to measure the correlation when Alice and Bob have their settings set at diagonal in polarization space, which corresponds to $\alpha=\beta=\pi / 8$ for the half-wave plates located in front of their respective PBS. Indeed, it is in the diagonal bases that the visibility of the correlation is naturally the lowest, and any departure from the optimal conditions reduces the absolute value of this correlation, whereas in the horizontal or vertical basis it is much less sensitive to imperfections. We have therefore measured the correlation in the diagonal bases while varying the temperature of the ppKTP crystal. The result is displayed in Fig. 3 (blue points).

At optimum temperature $\left(35.1{ }^{\circ} \mathrm{C}\right)$, the correlation in the diagonal bases $\alpha=\beta=\pi / 8$ is close to -1 . For small temperature variation of the ppKTP crystal away from the optimal temperature, the absolute value of the correlation in the diagonal bases decreases, which could be tempting to interpret as being caused by a loss of indistinguishability between the photons, as the centers of the spectral distributions of

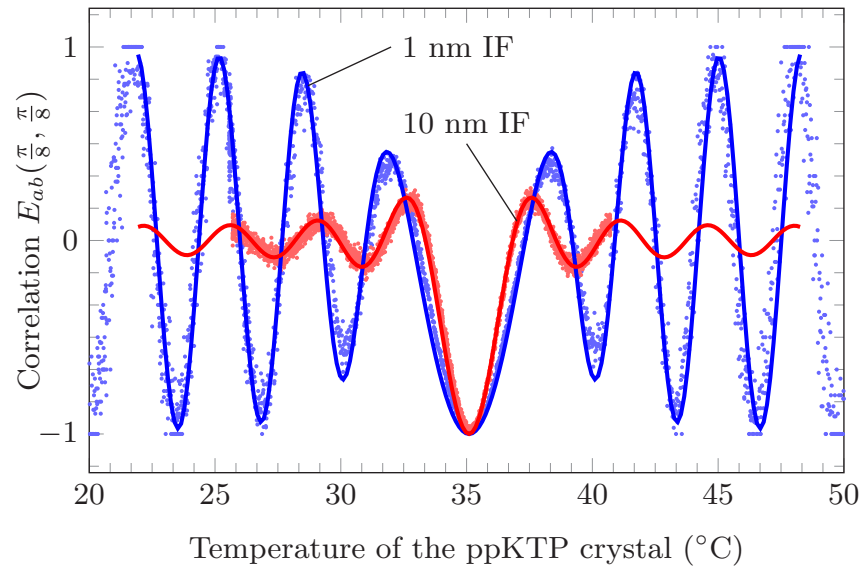

FIG. 3. Correlation in the diagonal bases $(\alpha=\beta=\pi / 8)$ as a function of temperature of the ppKTP crystal. Blue points are data acquired with a $1 \mathrm{~nm}$ IF while red points are acquired with a $10 \mathrm{~nm}$ IF. The corresponding curves are theoretical predictions. Although the rate of coincidences drops quickly away from the optimum temperature of $35.1{ }^{\circ} \mathrm{C}$, the correlation does not in any way disappear, but oscillates with increasing amplitudes. The occurrences of positive correlation close to 1 , indicating that the photons share the same polarization in the diagonal basis, are particularly worth investigating, given that we are operating in type II spontaneous parametric downconversion conditions, i.e., with orthogonally polarized photons.

the signal and idler photons start to differ more and more. However, the surprising feature revealed in Fig. 3 is that when departing further away from the optimum temperature, the correlation does not remain close to zero-as would be expected from distinguishable $H$ and $V$ photons observed in the diagonal bases-but oscillates instead with increasing amplitude, until the correlation reaches again absolute values close to unity.

Of particular interest are the temperature values for which the correlation becomes positive and close to 1 , which happens below the optimum temperature at 28.6, 25.0, and $21.8^{\circ} \mathrm{C}$. Indeed, it then means that the photons measured in the diagonal bases $(\alpha=\beta=\pi / 8)$ share the same polarization, which is surprising given that the downconverted photons are of type-II, that is, orthogonally polarized. We have observed that the orthogonality can actually still be seen quite clearly when Alice's and Bob's fix the orientation of their half-wave plates at $\alpha=\beta=0$ instead. The correlation is then very close to -1 .

It should be noted that these experimental features depend strongly on the use of the $1 \mathrm{~nm}$ bandwidth interference filter. When we instead used an interference filter with $10 \mathrm{~nm}$ bandwidth (Fig. 3, red points), the minima and maxima were shifted in temperature, and the amplitudes of the oscillations were greatly reduced, except at the optimum temperature where the correlation was still quite close to -1 . Indeed, the results are then very similar to those observed in [13] without any filter. Even if the experiment of Fedrizzi et al. is different in the setup relative to ours (separating the two polarization states and recombining them in a beamsplitter as in the original HOM experiment), it is closely related to our experiment in the analysis. In [13], positive correlation 
indicates that both photons exit the beamsplitter at different output ports. That is, it indicates photon antibunching, which implies a state that is antisymmetric in polarization.

The situation is different in our case because the photons are collinear and incident on the same input port |in $\rangle$ of the beamsplitter. The spatial modes of the idler and signal photons are also not distinguished in our experimental setup, so that it is a priori the same for both photons, and therefore symmetric by particle exchange. The compensation of the antisymmetry of the polarization degree of freedom is then not immediately obvious. As discussed in Ref. [13], one possible explanation comes from the fact that the photon state is frequency entangled. That is, each outgoing beam has a certain frequency spread, but the frequencies of the photons are not independently distributed over the frequency width of the beam. Rather, if one photon has a frequency higher than the center frequency of that beam, the other photon will have a frequency below the center frequency of the other beam, so that the energy conservation condition (3) is satisfied for each photon pair. As will be seen from the analysis below, our experiment, while different in setup, is logically completely equivalent to that in Ref. [13], except that we have used an additional interference filter to reduce the frequency spread of the two beams. Comparing the results with and without this filter, we make two interesting and initially surprising observations:

(i) The appearance of positive correlation, indicating antisymmetry of the polarization state, is explained by the presence of a corresponding antisymmetry in the frequencies, rendering the full state symmetric as expected for photons. That is, the observations are only explained if there is a certain frequency spread of the downconverted beams. Inserting a filter that limits this spread, one would expect the observed positive correlation to be reduced, whereas we observe the opposite. The correlation increases, reaching almost the maximum of +1 .

(ii) The perfect negative correlation at the optimal temperature is insensitive to the filter width. It appears to be the same for all possible frequency spreads.

In the following, we will address these issues and give detailed explanations of the observations.

\section{THEORETICAL DESCRIPTION}

\section{A. Spontaneous parametric downconversion}

The theoretical description of polarization entanglement from collinear type-II spontaneous parametric downconversion is well known (see, for instance, [6-12]). In a nonlinear crystal, the interaction Hamiltonian is [12]

$$
\hat{H}=\epsilon_{0} \int_{V} d r^{3} \chi^{(2)} \hat{E}_{p}^{+} \hat{E}_{1}^{-} \hat{E}_{2}^{-}+\text {H.c. }
$$

where the index $p$ stands for pump, while 1 and 2 refer to the two output modes (signal and idler). A photon from the pump can be spontaneously downconverted to two daughter photons. In a periodically poled crystal, this process must fulfill the quasi-phase-matching (QPM) conditions for the angular frequencies,

$$
\omega_{p}=\omega_{1}+\omega_{2},
$$

and for the wave numbers,

$$
k_{p}=k_{1}+k_{2}+\frac{2 \pi}{\Lambda},
$$

where $\Lambda$ is the period of the poling in the ppKTP crystal. For a crystal of finite length, Eq. (4) only has to be satisfied approximately, which results in a certain finite width of the spectral peak for the downconverted photons.

For collinear spontaneous parametric downconversion confined to a single spatial mode, the calculation to first-order perturbation theory for the quantum state of the pairs of downconverted photons at the output of the nonlinear crystal is $[20,21]$

$$
|\psi\rangle=W \int d v f(v) a_{\mathrm{in}, H}^{\dagger}\left(\omega_{1}\right) a_{\mathrm{in}, V}^{\dagger}\left(\omega_{2}\right)|0\rangle,
$$

where $a_{\mathrm{in}, H}^{\dagger}(\omega)$ and $a_{\mathrm{in}, V}^{\dagger}(\omega)$ are the creation operators for signal and idler photons in the input port |in $\rangle$ of the beamsplitter with horizontal and vertical polarization in frequency mode $\omega$, and where the integral is taken from $-\infty$ to $+\infty$. The function

$$
f(v)=\int_{-L}^{0} d z e^{i D z v}
$$

describes the spectral distribution of the downconverted photons. Here $L$ is the length of the ppKTP crystal, and we have assumed that it is oriented along the $z$-direction from $z=-L$ to $z=0$. We have defined

$$
D=\frac{1}{c_{2}}-\frac{1}{c_{1}},
$$

where $c_{1}$ and $c_{2}$ are the light speeds for the two output beams within the crystal. We let $\omega_{1}^{0}$ and $\omega_{2}^{0}$ be the output frequencies that satisfy both Eqs. (3) and (4), which means that they can be interpreted as the central frequencies of the spectral distributions of the two photon beams. The frequency deviation $v$ is then given by

$$
\omega_{1}=\omega_{1}^{0}+v, \quad \omega_{2}=\omega_{2}^{0}-v .
$$

\section{B. Simplified single-mode pair representation}

We start off with the theoretical description that can be found rather ubiquitously in the literature (see, for instance, [6-12]). As we will see, although this description will give the correct predictions at the optimal temperature of the ppKTP crystal, it will be insufficient at other temperatures.

Since we are selecting only the pairs that have a wavelength of $810 \mathrm{~nm}$ (with an interference filter with $1 \mathrm{~nm}$ bandwidth in our case), we can replace Eq. (5) by a single frequency mode, with two orthogonal polarizations $[6,7,12]$

$$
|\psi\rangle \propto a_{\mathrm{in}, H}^{\dagger}\left(\omega_{p} / 2\right) a_{\mathrm{in}, V}^{\dagger}\left(\omega_{p} / 2\right)|0\rangle=|H\rangle|V\rangle .
$$

To dispatch the photons to Alice and Bob, the pairs of collinear photons produced in the ppKTP crystal are sent to a nonpolarizing beamsplitter (NPBS). For a photon impinging with a spatial mode $\mid$ in $\rangle$ on an ideal 50:50 NPBS, the output state in terms of the transmitted mode $|a\rangle$ and the reflected mode $|b\rangle$ (sent, respectively, to Alice and Bob; see Fig. 1) 
depends on the initial polarization of the photon [6]:

$$
\begin{aligned}
& |H\rangle \mid \text { in }\rangle \stackrel{\text { NPBS }}{\longrightarrow} \frac{1}{\sqrt{2}}(|H\rangle|a\rangle+i|H\rangle|b\rangle), \\
& |V\rangle \mid \text { in }\rangle \stackrel{\text { NPBS }}{\longrightarrow} \frac{1}{\sqrt{2}}(|V\rangle|a\rangle-i|V\rangle|b\rangle),
\end{aligned}
$$

where the origin of the minus sign is due to the phase shift $\pi$ for a reflected wave with horizontal polarization at a beamsplitter.

If we now consider two orthogonally polarized photons impinging on the beamsplitter with the same input spatial mode, we can write, using the spatial mode as a shorthand index for the polarization mode,

$$
\begin{aligned}
|H\rangle_{\text {in }}|V\rangle_{\text {in }} \stackrel{\text { NPBS }}{\longrightarrow} & \frac{1}{2}\left(|H\rangle_{a}|V\rangle_{a}-i|H\rangle_{a}|V\rangle_{b}\right. \\
& \left.+i|H\rangle_{b}|V\rangle_{a}+|H\rangle_{b}|V\rangle_{b}\right) .
\end{aligned}
$$

The usual argument at this point [6-12] is that the cases in which the two photons exit through the same port (that is, $|H\rangle_{a}|V\rangle_{a}$ and $\left.|H\rangle_{b}|V\rangle_{b}\right)$ can be discarded because of the postselection of the photons. Only those pairs with one photon for Alice and one photon for Bob are of interest for the experimental results.

After making the substitution $|H\rangle_{b}|V\rangle_{a} \rightarrow|V\rangle_{a}|H\rangle_{b}$, and renormalizing, the state of the pairs of photons detected in coincidence by Alice and Bob can be written as the singlet state:

$$
\left|\Psi^{-}\right\rangle_{a b}=\frac{1}{\sqrt{2}}\left[|H\rangle_{a}|V\rangle_{b}-|V\rangle_{a}|H\rangle_{b}\right],
$$

which is a polarization-entangled state.

Starting from this postselected state, we want to calculate the predictions for the coincidence counting rates. The rotation imparted to the polarization of a photon by a half-wave plate oriented with an angle $\theta$ with respect to the horizontal can be written as

$$
\begin{aligned}
& |H\rangle \stackrel{\text { HWP } \theta}{\longrightarrow} \cos 2 \theta|H\rangle+\sin 2 \theta|V\rangle, \\
& |V\rangle \stackrel{\text { HWP } \theta}{\longrightarrow}-\sin 2 \theta|H\rangle+\cos 2 \theta|V\rangle .
\end{aligned}
$$

Using the transformations for the half-wave plate of Eqs. (13), with $\theta=\alpha$ for the photon going to Alice and $\theta=\beta$ for the photon going to Bob, the rates of coincidences measured by Alice and Bob take the simple and well-known form associated with the singlet state:

$$
\begin{aligned}
& R_{a b}^{++}=R_{a b}^{--} \propto \frac{1}{2} \sin ^{2} 2(\alpha-\beta), \\
& R_{a b}^{+-}=R_{a b}^{-+} \propto \frac{1}{2} \cos ^{2} 2(\alpha-\beta) .
\end{aligned}
$$

With good approximation, the rate of coincidences that we have measured at the optimal temperature are indeed of this form (see Fig. 4). The visibility (or contrast) of the coincidences was indeed slightly less than ideal, with a visibility of $99.6 \%$ in the rectilinear basis and of $98.5 \%$ in the diagonal basis (without subtraction of accidental coincidences).

In the above theory, the coincidence rates depend on the polarizers angles, but they are independent of the temperature. In particular, in the diagonal basis, $\alpha=\beta=\pi / 8$, we predict

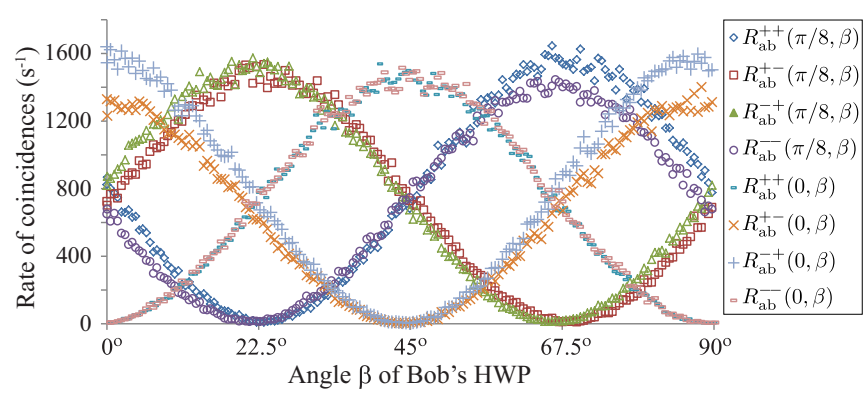

FIG. 4. Rates of coincidences in fixed bases while the temperature of the crystal is maintained at the optimum temperature of $35.1^{\circ} \mathrm{C}$. Alice keeps her measurement setting fixed, at $\alpha=0$ (rectilinear basis) or $\alpha=\pi / 8$ (diagonal basis), while Bob's HWP setting $\beta$ is varied from $0^{\circ}$ to $90^{\circ}$. The coincidences exhibit a visibility of $99.6 \%$ in the rectilinear basis and of $98.5 \%$ in the diagonal basis (without subtraction of accidental coincidences), which would amount to a $\mathrm{CHSH}$ function $S \simeq 2.80$.

$E_{a b}\left(\frac{\pi}{8}, \frac{\pi}{8}\right)=-1$ at all temperatures. As we see in Fig. 3, this is true only for the optimal temperature, and approximately true for a few other temperatures. Of particular interest are the temperature values for which the correlation becomes positive and close to 1 , as pointed out in detail above. We conclude that the single-mode pair representation fails at temperatures different from the optimal, and we proceed to give a more detailed analysis.

\section{Full theory using the frequency-entangled state}

We apply the conventional description (see, for example, Ref. [5]) for the propagation of the downconverted state, Eq. (5), through the optical elements. The details are given in Appendix. Here we only describe how to account for the effect of the interference filter, and we give the results for the two-photon amplitude.

When placing the interference filter in the beam, we have to modify the two-photon state of Eq. (5),

$$
|\psi\rangle=W \int d v f(v) F(v) a_{\mathrm{in}, H}^{\dagger}\left(\omega_{1}\right) a_{\mathrm{in}, V}^{\dagger}\left(\omega_{2}\right)|0\rangle,
$$

with the filter function $F(v)=\bar{G}\left(\omega_{1}\right) \bar{G}\left(\omega_{2}\right)$. The function $\bar{G}(\omega)$ describes the amplitude for a photon of frequency $\omega$ passing the filter. We assume that the function $\bar{G}$ has a peak centered on $\omega_{p} / 2$, so that we can write $\bar{G}(\omega)=G\left(\omega-\omega_{p} / 2\right)$ with the function $G(\omega)$ having a peak centered at zero. Defining $\mu=\omega_{1}^{0}-\omega_{2}^{0}$, Eq. (8) gives

$$
\omega_{1}=\frac{\omega_{p}}{2}+\frac{\mu}{2}+v, \quad \omega_{2}=\frac{\omega_{p}}{2}-\frac{\mu}{2}-v,
$$

and we get

$$
F(v)=G\left(\frac{\mu}{2}+v\right) G\left(\frac{-\mu}{2}-v\right) .
$$

We will restrict ourselves to symmetric filter functions, $G(-v)=G(v)$, and we have that

$$
F(v)=G\left(v+\frac{\mu}{2}\right)^{2} .
$$


To calculate the coincidence rates, we define the amplitude $\psi_{++}\left(t_{A}, t_{B}\right)$ for the detection of one photon in detector $A^{+}$at time $t_{A}$ and one in $B^{+}$at time $t_{B}$ :

$$
\psi_{++}\left(t_{A}, t_{B}\right)=\left\langle 0\left|E_{A+}^{(+)} E_{B+}^{(+)}\right| \psi\right\rangle
$$

with similar definitions for $\psi_{+-}, \psi_{-+}$, and $\psi_{--}$. Here $E_{A+}^{(+)}$ is the annihilation part of the field operator for the electric field at detector $A^{+}$, and similar for the other detectors. The exact form of these operators is given in Appendix, where it is shown that when $\alpha=\beta=\pi / 8$,

$$
\begin{aligned}
\psi_{++}\left(t_{B}, t_{A}\right)= & -\frac{W}{4} \int d v f(v) F(v) \\
& \times\left[e^{-i \omega_{1} \tau_{A+}^{H}-i \omega_{2} \tau_{B+}^{V}}-e^{-i \omega_{2} \tau_{A+}^{V}-i \omega_{1} \tau_{B+}^{H}}\right],
\end{aligned}
$$

where

$$
\begin{aligned}
\tau_{M \pm}^{H} & =t_{M \pm}-z_{M \pm} / c-\tau_{c} \\
\tau_{M \pm}^{V} & =t_{M \pm}-z_{M \pm} / c
\end{aligned}
$$

is the time a photon exits the ppKTP if it is detected at the detector $M \pm$ at time $t_{M \pm}\left(M=A, B\right.$ labels the detectors). $z_{M \pm}$ is the distance from the end of the ppKTP crystal to detector $M \pm$, and the time delay $\tau_{c}=L_{c} D$ (where $L_{c}$ is the length of the compensating crystal) is the difference in the time taken by photons with different polarizations to pass the compensating crystal.

The coincidence rate is then given by the standard expression (see, e.g., [5])

$$
R_{a b}^{++}=\frac{1}{2 T} \int_{0}^{2 T} d t_{+} \int d t_{-}\left|\psi_{++}\right|^{2},
$$

where $t_{ \pm}=t_{A} \pm t_{B}$. In the Appendix, we show that when $\alpha=$ $\beta=\pi / 8$, this gives

$$
\begin{aligned}
& R_{a b}^{++}=R_{a b}^{--}=R_{0}\left[I_{1}-I_{2}\right], \\
& R_{a b}^{+-}=R_{a b}^{-+}=R_{0}\left[I_{1}+I_{2}\right],
\end{aligned}
$$

with

$$
\begin{aligned}
& I_{1}=4 \int d \zeta \frac{G(\zeta+m / 2)^{4}}{\zeta^{2}} \sin ^{2} \frac{\zeta}{2} \\
& I_{2}=4 \int d \zeta \frac{G(\zeta+m / 2)^{4}}{\zeta(\zeta+m)} \sin \frac{\zeta}{2} \sin \frac{\zeta+m}{2}
\end{aligned}
$$

where we use the dimensionless variables

$$
\zeta=v D L, \quad m=\mu D L
$$

where we write $G(\omega)$ instead of $G(\omega / D L)$, and where we took the length $L_{c}$ of the compensating crystal to be half the length $L$ of the ppKTP crystal, as is the case in the experiment.

According to Eq. (1), the correlation in the diagonal basis is

$$
E_{a b}(\pi / 8, \pi / 8)=-\frac{I_{2}}{I_{1}} .
$$

The integrals $I_{1}$ and $I_{2}$ are probably difficult to calculate for most filter functions, and we are satisfied with numerical solutions of these integrals.

\section{Comparing with the experimental data}

To compare the theory with experiments, we need two things: the bandwidth of the interference filter and the scaling of the frequency difference between the two photons $\mu(T)$ with the temperature $T$.

We assume a Gaussian filter

$$
G(\zeta)=e^{-(\zeta / Z)^{2}}
$$

In the experiments, we used filters with nominal bandwidths of 1 and $10 \mathrm{~nm}$. For the $1 \mathrm{~nm}$ filter we measured the absorption of the filter spectroscopically and fitted a Gaussian of the form $e^{-\left(\frac{\lambda-\lambda_{0}}{W}\right)^{2}}$ with $\lambda_{0}=810 \mathrm{~nm}$. The average of two measurements gave a best fit $W=0.64 \mathrm{~nm}$, which is not far from the nominal specification. This has to be translated to the frequency-domain filter function (28). Since $G(\zeta)$ is the amplitude for the photon to pass the filter, the probability is $G(\zeta)^{2}$. Thus we set $2(\zeta / Z)^{2}=\left(\frac{\lambda-\lambda_{0}}{W}\right)^{2}$. Recalling that $\zeta=\Delta \omega D L$, where $\Delta \omega$ is the difference between the photon frequency and the center frequency of the filter, we have that

$$
\zeta=2 \pi D L c\left(\frac{1}{\lambda_{0}}-\frac{1}{\lambda}\right) \approx 2 \pi D L c \frac{\lambda-\lambda_{0}}{\lambda_{0}^{2}},
$$

where the approximation is valid as long as $\lambda-\lambda_{0} \ll \lambda_{0}$, which is appropriate for a narrow filter. Using that $D=$ $1 / c_{2}-1 / c_{1}=\left(n_{2}-n_{1}\right) / c$, where $n_{1}=1.75$ and $n_{2}=1.84$ are the indices of refraction of the two beams at the optimal temperature, we get

$$
Z=\frac{2 \sqrt{2} \pi\left(n_{2}-n_{1}\right) L}{\lambda_{0}^{2}} W=7.8,
$$

where $L=10 \mathrm{~mm}$ is the length of the ppKTP crystal. For the $10 \mathrm{~nm}$ filter, we do not have similar data but we can assume that it has an $A=80$, about 10 times as large as the $1 \mathrm{~nm}$ filter. In any case, it is so large that there will be no significant difference between the predicted result with this filter and with no filter at all.

To fully predict the experimental results, we also have to know the temperature dependence $\mu(T)$ of the difference in the center frequencies of the two downconverted beams. This can in principle be found from the phase-matching conditions (3) and (4), using the temperature dependence of the indices of refraction and the thermal expansion of the poling period. We tried several sets of published Sellmeier coefficients for KTP $[18,22,23]$, and they give substantially different results. None of them predict exactly the correct optimal temperature or a $\mu(T)$ that fits the results accurately. The optimal temperature is known from the data of Fig. 2, and it differs from the predicted value by a few degrees for the best sets of Sellmeier coefficients. The value of $\mu(T)$ is typically off by $10-50 \%$. We note that a similar situation is reported in Ref. [13]. It seems likely that the exact temperature dependence of the refraction indices is slightly different for different crystals, depending on the purity of the crystal and the growth and poling conditions. We have therefore used the measured value of 
the optimal temperature, and we assumed a linear dependence $\mu(T)=a\left(T-T_{\mathrm{opt}}\right)$ with the proportionality constant $a$ as a fitting parameter.

The results of the numerical evaluation of the integrals $I_{1}$ and $I_{2}$ with the corresponding filter functions give the correlation functions shown as lines in Fig. 3. The only fitting parameter is the proportionality constant $a$, and the same scaling is used for both filters. We see that the theory fully accounts for the experimental result. Observe that even with a $1 \mathrm{~nm}$ filter, the predictions are different from the simple single-frequency two-mode description discussed above. This means that the bandwidth of this filter is still too large for the simplified single-frequency two-mode description to be sufficient. It seems that the simplified description gives a satisfactory description only at the optimal temperature. We will now investigate why it works at this temperature, and how narrow the filters must be so that the results are the same as with a single-frequency mode.

\section{WHY THE TWO-MODE DESCRIPTION WORKS AT THE OPTIMAL TEMPERATURE}

\section{A. Without the interference filter}

Let us go back to Eq. (20) and insert the definition of $f(v)$. If we consider the case in which there is no interference filter, so that $F(v)=1$, we get (for simplicity, we write $\tau_{A}^{H}$ instead of $\tau_{A+}^{H}$ and similarly for all other quantities, it being understood that we consider the ++ correlation in these formulas)

$$
\begin{aligned}
\psi\left(t_{B}, t_{A}\right) \sim & \int_{-L}^{0} d z \int d v e^{i D z v} \\
& \times\left[e^{-i \omega_{1} \tau_{A}^{H}-i \omega_{2} \tau_{B}^{V}}-e^{-i \omega_{2} \tau_{A}^{V}-i \omega_{1} \tau_{B}^{H}}\right] .
\end{aligned}
$$

Here, $e^{-i \omega_{1} \tau_{A}^{H}}$ is the phase change of a horizontally polarized wave with frequency $\omega_{1}$ and wave velocity $c$ propagating from the origin (end of the ppKTP) to detector $A^{+}$, and similarly for the other phases. The factor $e^{i D z v}$ is due to the phase changes of two waves with frequencies $\omega_{1}$ and $\omega_{2}$ and wave velocities $c_{1}$ and $c_{2}$ from the point $z$ to the origin, multiplied by the phase $e^{i k_{p} z}$ of the pump field at point $z$. The interpretation of Eq. (31) is then that it is a sum of waves starting at all points $-L<z<0$ inside the ppKTP crystal and with all possible frequencies of the two outgoing waves, while keeping the energy conversion equation $\omega_{1}+\omega_{2}=\omega_{p}$. This is in the spirit of Feynman's path integral approach to quantum mechanics, where the total amplitude of a process is a sum over the amplitudes for all possible ways in which the process can take place.

We can give a more detailed geometric interpretation of this expression. Consider the first term in Eq. (31), which, as is well known [5,12], gives

$$
\int_{-L}^{0} d z \int d \nu e^{i D z v} e^{-i \omega_{1} \tau_{A}^{H}-i \omega_{2} \tau_{B}^{V}}=\Pi\left(\tau_{-} / D\right) e^{-i \omega_{1}^{0} \tau_{A}^{H}-i \omega_{2}^{0} \tau_{B}^{V}}
$$

where $\Pi(x)=1$ if $-L \leqslant x \leqslant 0$ and zero otherwise.

Recalling Eq. (21), we see that if we consider a fixed time, which we can choose as $t=0$, then $\tau_{M}^{P}$ (for detector
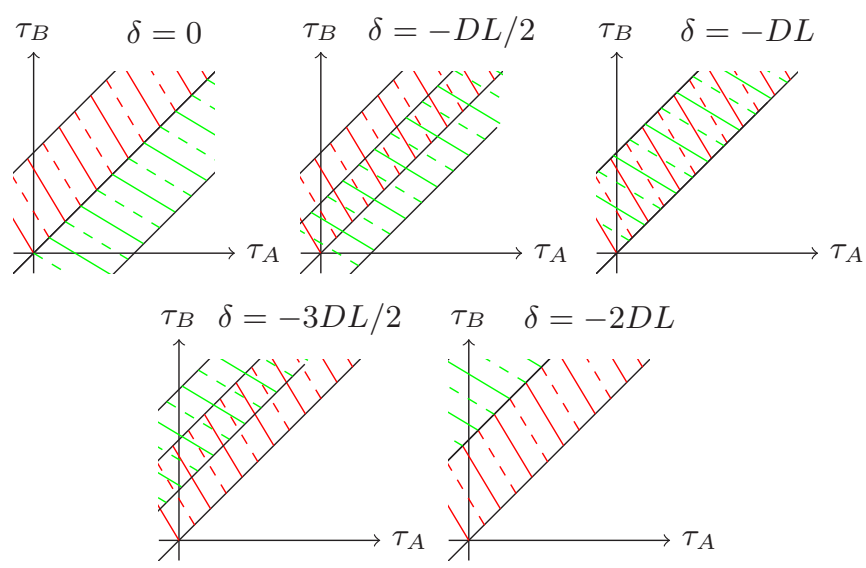

FIG. 5. Lines of equal phase for different thickness of the compensating crystal, represented by the different values of $\delta-2 \tau_{c}$. The compensating crystal used in the experiment corresponds to $\delta=$ $-D L$, which leads to maximal overlap of the wave bands. The lines of equal phase for the first term in Eq. (31) are shown in red, while those for the second term are green. To clarify the phase relations, we have shown a set of solid lines where the phase difference between each solid line is $2 \pi$. The dashed lines interspersed between the solid lines represent a phase shift of $\pi$ with respect to the solid lines. Outside of the two bands, the wave function is zero, as seen from Eq. (32), and two-photon interference only occurs when the two bands overlap.

$M=A, B$ and polarization $P=H, V)$ describes the changing phase of the wave in space along the given path. To get a full picture, we can plot the waves along the two paths along two orthogonal axes using the times $\tau_{M}^{P}$ as coordinates, and Eq. (32) describes a set of plane waves in this abstract space. As seen on the right-hand side of Eq. (32), the waves will interfere destructively outside of a certain band in the $\left(\tau_{A}, \tau_{B}\right)$ plane. Inside this band, we have a plane wave with the direction of the lines of equal phase (wavefronts) determined by the center frequencies $\omega_{1}^{0}$ and $\omega_{2}^{0}$. In the second term of Eq. (31), the detectors $A$ and $B$ are exchanged, so the image is reflected in the diagonal of the $\left(\tau_{A}, \tau_{B}\right)$ plane. In addition, there is a shift for finite $\delta=-2 \tau_{c}$ (where $\tau_{c}=L_{c} D$ is the difference between the times that the beams with orthogonal polarization use in passing the compensating crystal of length $L_{c}$ ):

$$
\begin{aligned}
\tau_{A}^{V} & =\tau_{A}^{H}-\delta / 2, \\
\tau_{B}^{H} & =\tau_{B}^{V}+\delta / 2,
\end{aligned}
$$

which gives the picture shown in Fig. 5, where the lines of equal phase from the first term of Eq. (31) are shown in red, while the lines of equal phase from the second term are green. Our experiments were performed with a compensating crystal whose length was half of the ppKTP crystal, which gives $\delta=$ $-D L$, corresponding to maximal overlap of the wave bands, and therefore maximal interference between the two-photon wave packets. From now on, we will only consider this value of $\delta$.

Consider now how this picture changes at different temperatures. As the temperature deviates from the optimal, the 

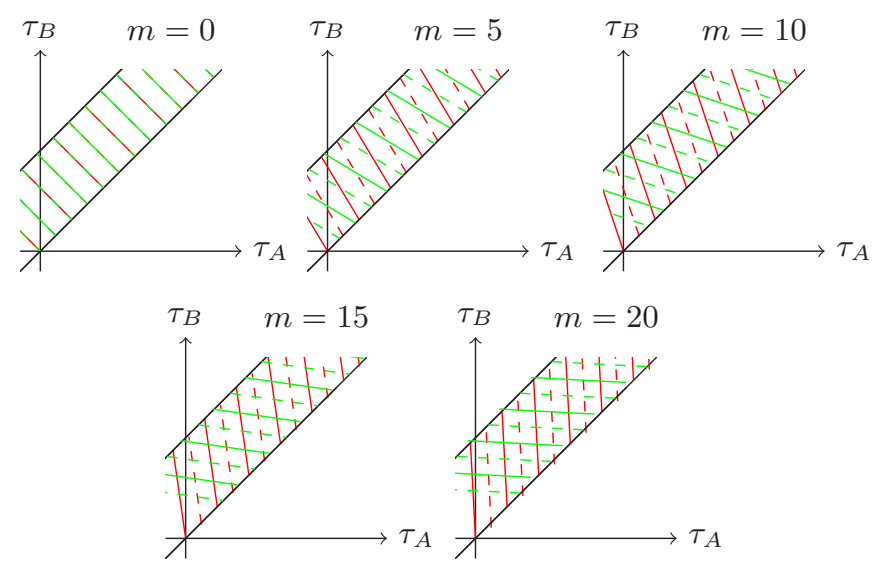

FIG. 6. Lines of equal phase for different temperatures, as represented by the value of $m=\mu(T) D L$, where $\mu(T)=\omega_{1}^{0}-\omega_{2}^{0}$ is the temperature-dependent difference between the center frequencies of the two beams. The case $m=0$ corresponds to the optimal temperature, while larger values of $m$ correspond to increasing deviations from the optimal temperature. All figures are for a compensating crystal with a length half that of the ppKTP as used in the experiments, which means that $\delta=-D L$ and that the bands from the two terms of Eq. (31) overlap fully, giving maximal two-photon interference. Colors are the same as in Fig. 5. As we see, for $m=0$, the two waves always meet in phase opposition, resulting in complete destructive interference at the center of the HOM dip.

center frequencies of the two outcoming beams start to differ, and the slope of the lines of equal phase changes. This is shown in Fig. 6 in terms of the dimensionless frequency difference $m$ as defined in Eq. (26). For $m=0$ the lines of equal phase for the two terms are parallel, and since there is a relative minus between the two terms, we get complete destructive interference, corresponding to the center of the HOM dip. This is the origin of the perfect anticorrelation, $E=$ -1 , observed at the optimal temperature. As the temperature deviates from the optimal, the lines of equal phase get tilted, and we get the oscillations in the correlation observed in Fig. 3 with the $10 \mathrm{~nm}$ IF (red points), which we check numerically is virtually indistinguishable from no IF. To understand how the oscillations of the correlation arise from the tilting of the lines of equal phase, we go back to Eq. (22), which gives the coincidence counting rate as an integral over the square of the two-photon amplitude (where we can change the integration variables to $\tau_{ \pm}=\tau_{A} \pm \tau_{B}$ ). The integrand will be independent of $\tau_{+}$, so that we can fix $\tau_{+}$at any value when considering the integral over $\tau_{-}$, which means integrating along a line crossing the wave band of Fig. 6. For $m=0$, the lines of equal phase for the two terms always meet in phase opposition, while if we look at $m=5$ we see that at the band edges the two terms meet in phase. However, to get the full picture, we have to remember that the wave function is a complex number, with real and imaginary parts. The coincidence rate is given by the integral in Eq. (22), and it is not so easy to visualize this complex behavior using the diagrams of Fig. 6. We will now see how this wave pattern is changed by the insertion of the IF, which limits the frequencies to a band around half the pump frequency.

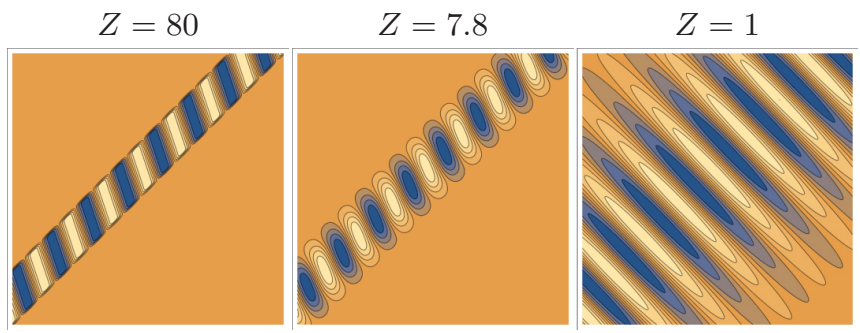

FIG. 7. Contour plots of the real part of the first term of Eq. (34) for different filter widths. The plots for $Z=80$ and 7.8 correspond to the experimental filters of 10 and $1 \mathrm{~nm}$, respectively. The plot for $Z=1$ corresponds to an even narrower filter than those used experimentally, and it is added to show the effect of the interference filter more clearly. As without the filter, the graphs for the second term of Eq. (34) are similar, just reflected in the diagonal and shifted according to the value of $\delta$.

\section{B. The effect of the interference filter}

With a filter in the setup, we have to go back to (15) and modify (31) accordingly (letting $\alpha=\beta=\pi / 8$ as before):

$$
\begin{aligned}
\psi\left(t_{B}, t_{A}\right) \sim & \int_{-L}^{0} d z \int d v F(v) e^{i D z v} \\
& \times\left[e^{-i \omega_{1} \tau_{A}^{H}-i \omega_{2} \tau_{B}^{V}}-e^{-i \omega_{2} \tau_{A}^{V}-i \omega_{1} \tau_{B}^{H}}\right] .
\end{aligned}
$$

As in (28), we choose a Gaussian filter function. The twophoton amplitude $\psi\left(t_{B}, t_{A}\right)$ is complex, so to visualize it we use contour plots of the real part as functions over the $\left(\tau_{A}, \tau_{B}\right)$ plane. In Fig. 7 we show the first term of Eq. (34) for different values of $Z$ and with $m=5$. As we decrease the filter bandwidth $Z$, two changes take place. First, the band where coincidences can occur widens and becomes less sharply defined. This is natural since all frequencies are needed to provide the sharp edges of the coincidence band (since a sharp step in a function only can be represented by a Fourier transform when all frequencies are included). Second, the lines of equal phase become more aligned $45^{\circ}$ to the axes.

We can now understand why the simple and popular singlefrequency two-mode description works at the optimal temperature, while it fails at other temperatures. At the optimal temperature, the lines of equal phase are parallel even without a filter. Adding a filter will smear out the band where the amplitude is nonzero, but it will not change the orientation of the lines of equal phase. So the interference effects between the two terms in Eq. (34) are unchanged as the filter width is changed. Only the overall amplitude, which is normalized away in the correlation function, is sensitive to the filter. Therefore, the results of the experiments are the same as if only one frequency was present, even if in the real experiment a superposition of frequencies occurs also at the optimal temperature. At temperatures different from the optimal temperature, the lines of equal phase are not parallel without the filter, and they are gradually made more parallel as the filter width is decreased. The experiments are then sensitive to the frequency spread of the downconverted photon beam, and the result depends on the filter width. 


\section{How narrow a filter do we need to have a single-frequency description?}

In the limit of a very narrow filter, we would still expect to recover the results of the one-frequency description. We can estimate how narrow a filter is needed at different temperatures using Eq. (25). If $G(\zeta)$ is a sharply peaked function around zero, we see that we will have contributions only for $\zeta$ close to $-\frac{m}{2}$. Then $\frac{\zeta}{2}=\frac{\zeta+m}{2}=\frac{m}{4}$, and both integrals will be close to zero when $m=4 \pi n$. We are interested in $m$ close to these points. We let $\zeta=-m / 2+\xi$ and expand for small $\xi$ :

$$
\begin{aligned}
f_{1}= & \frac{\sin ^{2} \frac{\zeta}{2}}{\zeta^{2}}=\frac{4 \sin ^{2} m / 2}{m^{2}}-(\cdots) \xi \\
& +\frac{m^{2} \cos m / 2+48 \sin ^{2} m / 4-8 m \sin m / 2}{m^{4}} \xi^{2}, \\
f_{2}= & \frac{\sin \frac{\zeta}{2} \sin \frac{\zeta+m}{2}}{\zeta(\zeta+m)}=\frac{4 \sin ^{2} m / 2}{m^{2}}-\frac{m^{2}-16 \sin ^{2} m / 4}{m^{4}} \xi^{2},
\end{aligned}
$$

where the linear term in $f_{1}$ is unimportant since it will disappear when integrated over a symmetric filter function $G(\zeta)$. When $m$ is close to $4 \pi n$ (and $n \neq 0$ ) we can ignore the variations of the quadratic coefficients with $m$ and set $m=4 \pi n$ in the trigonometric functions to get

$$
\begin{aligned}
& f_{1}=\frac{4 \sin ^{2} \Delta m / 2}{m^{2}}+\frac{\xi^{2}}{m^{2}}, \\
& f_{2}=\frac{4 \sin ^{2} \Delta m / 2}{m^{2}}-\frac{\xi^{2}}{m^{2}},
\end{aligned}
$$

where $\Delta m=m-4 \pi n$. For simplicity, assume a sharp filter function $G(\zeta)=1$ when $|\zeta|<Z$ and $G(\zeta)=0$ when $|\zeta|>$ $Z$. Then

$$
I_{1,2}=\frac{8 Z}{3 \Delta m^{2}}\left(12 \sin ^{2} \frac{\Delta m}{4} \pm Z^{2}\right),
$$

where the upper sign is for $I_{1}$ and the lower sign is for $I_{2}$. We see that if $Z>3 \sqrt{2} \sin \frac{\Delta m}{4} \approx \Delta m$, the second term dominates, and $I_{2}=-I_{1}$, which gives a correlation $E_{A B}=$ $-\frac{I_{2}}{I_{1}}=+1$, meaning that the outcome at Alice's arm is equal to that at Bob's arm. If $Z<\Delta m$, we have $I_{2}=I_{1}$, which gives a correlation $E_{A B}=-1$, meaning that the outcome at Alice's arm is opposite to that at Bob's arm. Looking back to the experiments in Sec. III we see that the introduction of a narrower filter increased the amplitude of the oscillations of the correlation, and made the correlation closer to +1 . At the same time, we now see that a certain spread in frequency is necessary for the change between a correlation -1 and +1 , and that with a sufficiently narrow filter, we expect the correlation to go back to -1 . For $m$ close to $4 \pi n$ we then expect the correlation to depend nonmonotonically on the filter width $Z$.

\section{SUMMARY AND DISCUSSION}

We have shown the results of a two-photon interference experiment in which entangled pairs of photons are created by parametric downconversion in a ppKTP crystal. The downconversion process is most efficient at a certain optimal temperature, and we have shown the effect of changing the temperature away from this optimum. In previous experiments [13] it had been shown that the temperature dependence of the results reveals the frequency entanglement of the two-photon state, as described by Eq. (5). This had been observed through the presence of a slightly positive value of the correlation function (1) between the two-photon detections.

We have demonstrated the same effect in a different setup, and we have found dramatic oscillations of the correlation between -1 and +1 as the temperature was changed. This happened in an experimental system where the two photon beams are never recombined in a beamsplitter (after the primary nonpolarizing beamsplitter), but where the two polarization states are mixed using half-wave plates. In addition, we have investigated the effect of inserting an interference filter before the two beams are separated by a beamsplitter to reduce the frequency window over which the photons are spread.

The most natural expectation is then that the presence of a filter will reduce the effect of the frequency entanglement, and thereby also the positive value of Eq. (1). This is also the result of a simplified theoretical description that ignores the frequency spread and includes only a single frequency mode. We observe the opposite, in the sense that the correlation comes close to the maximal value of +1 at certain temperatures, as seen in Fig. 3.

We have provided a detailed theoretical analysis, which shows excellent agreement with the experiments. We have also given an interpretation of the analytical formulas in terms of the representation of the two-photon amplitude in the space spanned by the detection times of the two photons.

Our work demonstrates that the simplified single-mode pair representation is insufficient to describe our experimental results. The biphoton description involves a much more complicated interplay between the two original photons created "simultaneously" in the nonlinear crystal. The interplay involves in a very detailed manner both frequency relations between the two original photons and timing differences (phase shifts) on their way from where they are created until the horizontal and vertically polarized contributions have passed through the rest of the nonlinear crystal, as well as through the compensating crystal, the filters, and the mixing NPBS. All these details are contained in the biphoton state.

\section{APPENDIX: DETAILS OF THE CALCULATION OF THE TWO-PHOTON AMPLITUDE AND COINCIDENCE RATE}

We let operators $a_{a, H}$ and similar represent the field after the NPBS but before the HWP, and $a_{A,+}$ and similar after the HWP. The effect of the HWP is then described by the transformation

$$
\begin{aligned}
& a_{A,+}=\cos 2 \alpha a_{a, H}+\sin 2 \alpha a_{a, V}, \\
& a_{A,-}=-\sin 2 \alpha a_{a, H}+\cos 2 \alpha a_{a, V}, \\
& a_{B,+}=\cos 2 \beta a_{b, H}+\sin 2 \beta a_{b, V}, \\
& a_{B,-}=-\sin 2 \beta a_{b, H}+\cos 2 \beta a_{b, V} .
\end{aligned}
$$

The effect of the PBS is to send the two different polarizations to the two different detectors, so that the field at one of the 
detectors is

$$
E_{A,+}^{(+)}=i \int d \omega_{A} A\left(\omega_{A}\right) e^{-i \omega_{A} \tau_{A+}^{P}} a_{A,+}\left(\omega_{A}\right)
$$

and similarly for the others. Here the time $\tau_{A+}^{P}$ depends on the initial polarization $P$ of the photon hitting the detector, as will be specified below. The action of the NPBS is to split each photon into the two arms, with extra phase factors in some cases according to

$$
\begin{aligned}
& a_{\mathrm{in}, H} \stackrel{\mathrm{NPBS}}{\longrightarrow} \frac{1}{\sqrt{2}}\left(a_{a, H}+i a_{b, H}\right), \\
& a_{\mathrm{in}, V} \stackrel{\mathrm{NPBS}}{\longrightarrow} \frac{1}{\sqrt{2}}\left(a_{a, V}-i a_{b, V}\right) .
\end{aligned}
$$

This gives the field operators at the detectors in terms of the operators at the exit of the ppKTP:

$$
\begin{aligned}
& E_{A+}^{(+)}=\frac{i}{\sqrt{2}} \int d \omega_{A} A\left(\omega_{A}\right)\left[e^{-i \omega_{A} \tau_{A+}^{H}} \cos 2 \alpha a_{\mathrm{in}, H}+e^{-i \omega_{A} \tau_{A+}^{V}} \sin 2 \alpha a_{\mathrm{in}, V}\right], \\
& E_{A-}^{(+)}=\frac{i}{\sqrt{2}} \int d \omega_{A} A\left(\omega_{A}\right)\left[-e^{-i \omega_{A} \tau_{A-}^{H}} \sin 2 \alpha a_{\mathrm{in}, H}+e^{-i \omega_{A} \tau_{A-}^{V}} \cos 2 \alpha a_{\mathrm{in}, V}\right], \\
& E_{B+}^{(+)}=\frac{1}{\sqrt{2}} \int d \omega_{B} A\left(\omega_{B}\right)\left[e^{-i \omega_{B} \tau_{B+}^{H}} \cos 2 \beta a_{\mathrm{in}, H}-e^{-i \omega_{B} \tau_{B+}^{V}} \sin 2 \beta a_{\mathrm{in}, V}\right], \\
& E_{B-}^{(+)}=\frac{1}{\sqrt{2}} \int d \omega_{B} A\left(\omega_{B}\right)\left[-e^{-i \omega_{B} \tau_{B-}^{H}} \sin 2 \beta a_{\mathrm{in}, H}-e^{-i \omega_{B} \tau_{B-}^{V}} \cos 2 \beta a_{\mathrm{in}, V}\right] .
\end{aligned}
$$

Here

$$
\tau_{M \pm}^{H}=t_{M \pm}-z_{M \pm} / c-\tau_{c}, \quad \tau_{M \pm}^{V}=t_{M \pm}-z_{M \pm} / c
$$

is the relative time a photon exited the ppKTP if it is detected at the detector $M \pm$ at time $t_{M \pm}(M=A, B$ labels the detectors). The time delay $\tau_{c}=L_{c} D$ (where $L_{c}$ is the length of the compensating crystal) is the difference in the time it takes for photons with different polarizations to pass the compensating crystal.

Using this [and referring to Eqs. (6) and (15)], we get

$$
\begin{aligned}
\psi_{++}\left(t_{A}, t_{B}\right)= & \left\langle 0\left|E_{A+}^{(+)} E_{B+}^{(+)}\right| \psi\right\rangle \\
= & -\frac{W}{2} \int d \omega_{A} d \omega_{B} d \nu f(v) F(v)\langle 0|\left[e^{-i \omega_{A} \tau_{A+}^{H}} \cos 2 \alpha a_{\mathrm{in}, H}\left(\omega_{A}\right)+i e^{-i \omega_{A} \tau_{A+}^{V}} \sin 2 \alpha a_{\mathrm{in}, V}\left(\omega_{A}\right)\right] \\
& \times\left[i e^{-i \omega_{B} \tau_{B+}^{H}} \cos 2 \beta a_{i n, H}\left(\omega_{B}\right)+e^{-i \omega_{B} \tau_{B+}^{V}} \sin 2 \beta a_{\mathrm{in}, V}\left(\omega_{B}\right)\right] a_{\mathrm{in}, H}^{\dagger}\left(\omega_{1}\right) a_{\mathrm{in}, V}^{\dagger}\left(\omega_{2}\right)|0\rangle \\
= & -\frac{W}{2} \int d \omega_{A} d \omega_{B} d \nu f(\nu) F(v)\left[e^{-i \omega_{A} \tau_{A+}^{H}-i \omega_{B} \tau_{B+}^{V}} \cos 2 \alpha \sin 2 \beta \delta\left(\omega_{A}-\omega_{1}\right) \delta\left(\omega_{B}-\omega_{2}\right)\right. \\
& \left.-e^{-i \omega_{A} \tau_{A+}^{V}-i \omega_{B} \tau_{B+}^{H}} \sin 2 \alpha \cos 2 \beta \delta\left(\omega_{A}-\omega_{2}\right) \delta\left(\omega_{B}-\omega_{1}\right)\right] \\
= & -\frac{W}{2} \int d \nu f(\nu) F(v)\left[e^{-i \omega_{1} \tau_{A}^{H}-i \omega_{2} \tau_{B}^{V}} \cos 2 \alpha \sin 2 \beta-e^{-i \omega_{2} \tau_{A}^{V}-i \omega_{1} \tau_{B}^{H}} \sin 2 \alpha \cos 2 \beta\right] .
\end{aligned}
$$

Similarly, we find that

$$
\psi_{+-}\left(t_{A}, t_{B}\right)=-\frac{W}{2} \int d \nu f(\nu) F(\nu)\left[e^{-i \omega_{1} \tau_{A}^{H}-i \omega_{2} \tau_{B}^{V}} \cos 2 \alpha \cos 2 \beta+e^{-i \omega_{2} \tau_{A}^{V}-i \omega_{1} \tau_{B}^{H}} \sin 2 \alpha \sin 2 \beta\right],
$$

which for $\alpha=\beta=\pi / 8$ (and we write the following equations only in this case) is the same as $\psi_{++}$except for the sign of the second term. The remaining $\psi_{--}$and $\psi_{-+}$are related to the ones given by simple symmetry relations.

Defining $\tau_{ \pm}=\tau_{A}^{H}-\tau_{B}^{V}$, we can rewrite Eq. (A6) for $\alpha=\beta=\pi / 8$ as [see also Eqs. (16)-(18) and (33)]

$$
\psi_{++}\left(t_{A}, t_{B}\right)=-\frac{W}{2} \int d v f(v) F(v) e^{-i \frac{\omega_{p}}{2} \tau_{+}}\left[e^{-i\left(\frac{\mu}{2}+v\right) \tau_{-}}-e^{i\left(\frac{\mu}{2}+v\right)\left(\tau_{-}-\delta\right)}\right] .
$$

For the coincidence rate, we then find

$$
\begin{aligned}
R_{a b}^{++}= & \frac{D^{2} R_{0}}{2} \int d \tau_{-} \int d v_{1} d v_{2} \int_{-L}^{0} d z_{1} d z_{2} e^{i D\left(z_{2} v_{2}-z_{1} v_{1}\right)} \\
& \times\left[e^{i\left(v_{1}-v_{2}\right) \tau_{-}}+e^{i\left(v_{2}-v_{1}\right)\left(\tau_{-}-\delta\right)}-e^{i\left(\mu+v_{1}+v_{2}\right) \tau_{-}-\frac{i}{2} \mu \delta-i v_{2} \delta}-e^{-i\left(\mu+v_{1}+v_{2}\right) \tau_{-}+\frac{i}{2} \mu \delta+i v_{1} \delta}\right] F\left(v_{1}\right) F\left(v_{2}\right) \\
= & \frac{D^{2} R_{0}}{2} \int d \nu_{1} d v_{2} \int_{-L}^{0} d z_{1} d z_{2} e^{i D\left(z_{2} v_{2}-z_{1} v_{1}\right)} \\
& \times\left[2 \delta\left(v_{1}-v_{2}\right)-\delta\left(\mu+v_{1}+v_{2}\right)\left(e^{-\frac{i}{2} \mu \delta-i v_{2} \delta}+e^{\frac{i}{2} \mu \delta+i v_{1} \delta}\right)\right] F\left(v_{1}\right) F\left(v_{2}\right)
\end{aligned}
$$




$$
\begin{aligned}
& =D^{2} R_{0} \int d v \int_{-L}^{0} d z_{1} d z_{2}\left[F(v)^{2} e^{i D\left(z_{2}-z_{1}\right) v}-F(v) F(-\mu-v) e^{i D\left(z_{1}+z_{2}\right) v+i D z_{1} \mu} e^{-\frac{i}{2} \mu \delta-i v \delta}\right] \\
& =R_{0} \int d v\left[\frac{F(v)^{2}}{v^{2}}\left[1-e^{-i D L v}\right]\left[1-e^{i D L v}\right]+\frac{F(v) F(-\mu-v)}{v(\mu+v)}\left[1-e^{-i D L(v+\mu)}\right]\left[1-e^{-i D L v}\right] e^{-\frac{i}{2} \mu \delta-i v \delta}\right],
\end{aligned}
$$

which gives Eqs. (23)-(25) of the main text when the length $L_{c}$ of the compensating crystal is half the length $L$ of the ppKTP crystal.

[1] C. K. Hong, Z. Y. Ou, and L. Mandel, Phys. Rev. Lett. 59, 2044 (1987).

[2] T. B. Pittman, D. V. Strekalov, A. Migdall, M. H. Rubin, A. V. Sergienko, and Y. H. Shih, Phys. Rev. Lett. 77, 1917 (1996).

[3] Y.-H. Kim and W. P. Grice, J. Opt. Soc. Am. B 22, 493 (2005).

[4] A. V. Sergienko, Y. H. Shih, and M. H. Rubin, J. Opt. Soc. Am. B 12, 859 (1995).

[5] Y. Shih, An Introduction to Quantum Optics: Photon and Biphoton Physics (Taylor \& Francis, London, 2011).

[6] T. Suhara, Las. Photon. Rev. 3, 370 (2009).

[7] T. Suhara, H. Okabe, and M. Fujimura, IEEE Photon. Tech. Lett. 19, 1093 (2007).

[8] A. Martin, V. Cristofori, P. Aboussouan, H. Herrmann, W. Sohler, D. B. Ostrowsky, O. Alibart, and S. Tanzilli, Opt. Exp. 17, 1033 (2009).

[9] A. Martin, A. Issautier, H. Herrmann, W. Sohler, D. B. Ostrowsky, O. Alibart, and S. Tanzilli, New J. Phys. 12, 103005 (2010).

[10] C. E. Kuklewicz, Ph.D. thesis, Massachusetts Institute of Technology, 2005.

[11] F. Sciarrino, G. Vallone, A. Cabello, and P. Mataloni, Phys. Rev. A 83, 032112 (2011).
[12] M. H. Rubin, D. N. Klyshko, Y. H. Shih, and A. V. Sergienko, Phys. Rev. A. 50, 5122 (1994).

[13] A. Fedrizzi, T. Herbst, M. Aspelmeyer, M. Barbieri, T. Jennewein, and A. Zeilinger, New J. Phys. 11, 103052 (2009).

[14] G. Brida, M. Chekhova, M. Genovese, and L. Krivitsky, Phys. Rev. A 76, 053807 (2007).

[15] G. Adenier, J. Bergli, A. P. Thörn, and A. I. Vistnes, Proc. SPIE 8832, 88321K (2013).

[16] C. E. Kuklewicz, M. Fiorentino, G. Messin, F. N. C. Wong, and J. H. Shapiro, Phys. Rev. A 69, 013807 (2004).

[17] G. Weihs, T. Jennewein, C. Simon, H. Weinfurter, and A. Zeilinger, Phys. Rev. Lett. 81, 5039 (1998).

[18] S. Emanueli and A. Arie, Appl. Opt. 42, 6661 (2003).

[19] D. R. Hamel, M. Sc. thesis, Waterloo University, Canada, 2010.

[20] M. Atatüre, G. Di Giuseppe, M. D. Shaw, A. V. Sergienko, B. E. A. Saleh, and M. C. Teich, Phys. Rev. A 66, 023822 (2002).

[21] G. DiGiuseppe, M. Atature, M. D. Shaw, A. V. Sergienko, B. E. A. Saleh, M. C. Teich, A. J. Miller, S. W. Nam, and J. Martinis, Phys. Rev. A 68, 063817 (2003).

[22] K. Kato and E. Takaoka, Appl. Opt. 41, 5040 (2002).

[23] H. Zhao, I. T. Lima Jr., and A. Major, Proc. SPIE 7750, 77501D1 (2010). 